\title{
Patients with computed tomography-proven acute diverticulitis require follow-up to exclude colorectal
}

\section{cancer}

\author{
Shafquat Zaman ${ }^{1}$, Warren Chapman ${ }^{2}$, Imtiyaz Mohammed ${ }^{3}$, Kathryn Gill ${ }^{1}$, Stephen Thomas Ward ${ }^{4}$ \\ ${ }^{I}$ Department of General Surgery, Sandwell \& West Birmingham Hospitals NHS Trust, Sandwell General Hospital, West Bromwich, \\ ${ }^{2}$ Nurse Consultant and Clinical Lead for Endoscopy at City Hospital Endoscopy Unit, Sandwell \& West Birmingham Hospitals NHS Trust, \\ Birmingham City Hospital, Birmingham, ${ }^{3}$ Department of Gastroenterology, Sandwell \& West Birmingham Hospitals NHS Trust, Sandwell \\ General Hospital, West Bromwich, ${ }^{4}$ Department of General Surgery, University Hospitals Coventry \& Warwickshire NHS Trust, Walsgrave, UK
}

\begin{abstract}
Background/Aims: Traditionally, patients with acute diverticulitis undergo follow-up endoscopy to exclude colorectal cancer (CRC). However, its usefulness has been debated in this era of high-resolution computed tomography (CT) diagnosis. We assessed the frequency and outcome of endoscopic follow-up for patients with CT-proven acute diverticulitis, according to the confidence in the CT diagnosis. Methods: Records of patients with CT-proven acute diverticulitis between October 2007 and March 2014 at Sandwell \& West Birmingham Hospitals NHS Trust were retrieved. The National Cancer Registry confirmed the cases of CRC. Endoscopy quality indicators were compared between these patients and other patients undergoing the same endoscopic examination over the same period. Results: We identified 235 patients with CT-proven acute diverticulitis, of which, 187 were managed conservatively. The CT report was confident of the diagnosis of acute diverticulitis in $75 \%$ cases. Five of the 235 patients were subsequently diagnosed with CRC (2.1\%). Three cases of CRC were detected in the 187 patients managed conservatively (1.6\%). Forty-eight percent of the conservatively managed patients underwent follow-up endoscopy; one case of CRC was identified. Endoscopies were often incomplete and caused more discomfort for patients with diverticulitis compared with controls. Conclusions: CRC was diagnosed in patients with CT-proven diverticulitis at a higher rate than in screened asymptomatic populations, necessitating follow-up. CT reports contained statements regarding diagnostic uncertainty in $25 \%$ cases, associated with an increased risk of CRC. Follow-up endoscopy in patients with CT-proven diverticulitis is associated with increased discomfort and high rates of incompletion. The use of other follow-up modalities should be considered. (Intest Res 2017;15:195-202)
\end{abstract}

Key Words: Diverticulitis; Follow-up; Endoscopy; Colorectal neoplasms

\section{INTRODUCTION}

Diverticula can occur throughout the length of the gastrointestinal tract but are most commonly seen in the sigmoid

Received August 16, 2016. Revised October 21, 2016. Accepted October 27, 2016. Published online March 17, 2017 Correspondence to Stephen Thomas Ward, Department of General Surgery, University Hospitals Coventry \& Warwickshire NHS Trust, Clifford Bridge Road, Walsgrave, West Midlands CV3 2DX, UK. Tel: +44-2476-966102,

Fax: +44-2476-966090, E-mail: stephen.ward7@nhs.net

Financial support: None. Conflict of interest: None. and descending colon and become increasingly more common with age. In the United Kingdom, an estimated 50\% of the population over the age of 50 years are affected and this proportion increases to almost $70 \%$ by the age of 80 years. ${ }^{1}$ The majority of those affected remain asymptomatic but presentations can vary from mild predominantly left-sided abdominal pain through to a perforated bowel. ${ }^{2}$

A number of risk factors are thought to be involved with the development of diverticular disease including a low dietary fiber intake, lack of exercise, smoking, obesity and immunosuppression. ${ }^{2} \mathrm{~A}$ low fiber diet is associated with the

\footnotetext{
(c) Copyright 2017. Korean Association for the Study of Intestinal Diseases. All rights reserved.

This is an Open Access article distributed under the terms of the Creative Commons Attribution Non-Commercial License (http://creativecommons.org/licenses/by-nc/4.0)

which permits unrestricted non-commercial use, distribution, and reproduction in any medium, provided the original work is properly cited.
} 
formation of small volume stools that require high intraluminal pressures for propulsion. It is thought that this may be a mechanism leading to the formation of diverticula.

Acute diverticulitis occurs in an estimated $10 \%$ to $25 \%$ of patients with diverticulosis. Complications of diverticulitis include abscess formation, perforation, fistulation, obstruction, and hemorrhage. The differential diagnoses that need to be considered in these patients include malignancy, colitis, and IBS. ${ }^{3}$ Not all patients with diverticulitis require hospital admission but more severe cases require intravenous antibiotics and/or emergency surgery. ${ }^{4}$

Endoscopic imaging of the colon is generally avoided in patients during an attack of acute diverticulitis because of the perceived risk of bowel perforation. The traditional management of diverticulitis requires mandatory endoscopic examination of the colon following resolution of the acute episode in order to exclude colorectal cancer (CRC). Commissioning guidelines published by the Royal College of Surgeons (2014) state that "all patients require investigation of the colonic lumen by endoscopy, barium enema or CT colonography after the acute attack has resolved." ${ }^{\prime 4}$ This is typically deferred for 4 to 6 weeks. The position statement from the Association of Coloproctology of Great Britain and Ireland is similar in its recommendations. It states that "the diagnosis of diverticulitis should be confirmed during the acute attack by radiological means. The modality should be CT or ultrasound depending on local expertise. Barium enema or colonoscopy after resolution of the acute episode is essential to rule out alternative diagnoses or second pathologies." ${ }^{3}$ The recently revised guidelines (2014) from the American Society of Colon and Rectal Surgeons recommend colonoscopy or CT colonography to evaluate the colon 6 to 8 weeks after recovery from acute diverticulitis. ${ }^{5}$

Recent studies have questioned this approach given the low diagnostic yield of endoscopy following CT-proven diverticulitis. ${ }^{6-9}$ The main objective of this study was to determine if all patients with CT-proven diverticulitis necessarily need further evaluation by endoscopy or whether these investigations could be reserved for a selected subgroup of patients alone, based on the confidence of the CT report.

\section{METHODS}

\section{Defining the Patient Population}

The picture archiving and communication system (PACS) database was interrogated to identify all patients who underwent an abdominal CT scan between October 2007 and
March 2014 at the Sandwell \& West Birmingham Hospitals NHS Trust, the report of which contained the term "diverticulitis." The reports were then manually checked to identify those reporting acute diverticulitis. This defined a group of patients with CT-proven diverticulitis. The CT report was defined as possessing diagnostic uncertainty if the report stated that CRC was a possible differential diagnosis or if the report recommended confirmatory endoscopic examination. All CT scans prior to March 2013 were performed using a 16-slice Somatom Sensation scanner (Siemens, Munich, Germany); the majority after this date were performed using the 128-slice Flash CT scanner (Siemens). All scans were reported by radiologists with a specialist interest in CT, although not necessarily an interest in gastrointestinal CT.

\section{Follow-Up and Outcome Data}

Hospital case notes and endoscopy reporting software were used to determine which patients underwent a lower gastrointestinal endoscopy, either colonoscopy or flexible sigmoidoscopy, following their CT-based diagnosis. The extent of the endoscopic examination, endoscopist assessment of discomfort, duration of the endoscopy and endoscopic findings were recorded. In terms of endoscopic findings, the presence or absence of diverticulosis, strictures, IBD, polyps, and CRC were noted. The National Cancer Registry was interrogated using the Cancer Analysis System (National Cancer Registration Service, Public Health England) to identify cases of CRC from our cohort of patients with CT-proven diverticulitis.

The management of each patient and length of hospital stay was determined from hospital case notes. The management was classified as conservative (antibiotics), radiologicallyguided drainage, laparoscopic washout, or sigmoid resection.

\section{Endoscopy Quality Indicators}

Data for all colonoscopy and flexible sigmoidoscopy procedures performed at the Trust over the same time period were retrieved from the local endoscopy reporting software (ADAM; Fujinon, Saitama, Japan). The data fields retrieved were: the extent of the examination, endoscopist assessment of discomfort, and duration of the procedure. All endoscopies were performed or overseen by Joint Advisory Group approved endoscopists. A colonoscopy was defined as complete if the examination reached the terminal ileum, neoterminal ileum, surgical anastomosis, or cecum. A flexible sigmoidoscopy was defined as complete if the examination 
reached the descending colon or a more proximal location. Discomfort was not a mandatory field and was assessed as none, mild, moderate, or severe. The duration of the procedure in minutes was not a mandatory field. The extent, discomfort, and duration of lower gastrointestinal endoscopies were compared between patients with CT-proven diverticulitis (cases) and all other endoscopies of the same type over the same time period. Differences in extent and discomfort were tested for statistical significance by the chi-square test, and for duration by the Mann-Whitney test. All statistical calculations were performed using $\mathrm{R}$ version 2.15.2 (The $\mathrm{R}$ Foundation for Statistical Computing, Vienna, Austria). Calculated values were reported to two significant figures and $P$-values $<0.05$ were considered statistically significant.

Approval for this project was given by the local NHS Trust Clinical Governance department and Public Health England. Data were anonymized and destroyed after use.

\section{RESULTS}

\section{Patient Demographics}

Interrogation of the PACS database, CT reports, and hos-

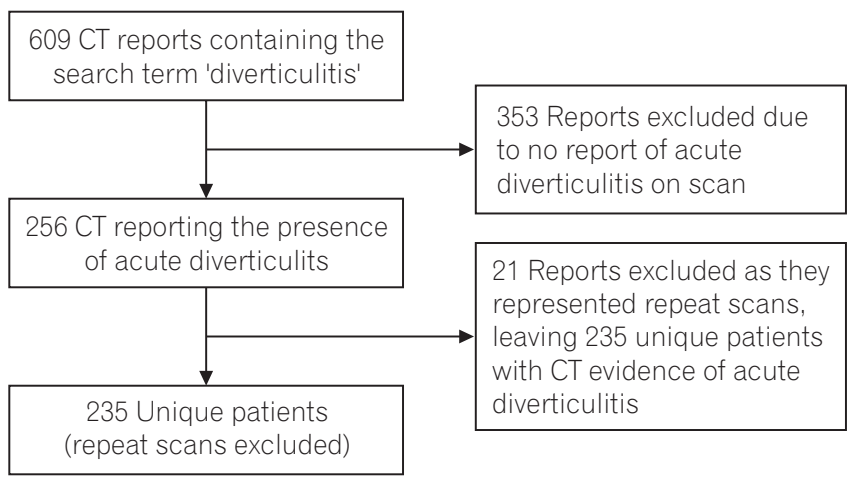

Fig. 1. Study protocol. pital notes identified 235 patients (109 male, 126 female) with CT-proven diverticulitis (Fig. 1). The median age was 69 years (IQR, 55-79; range, 35-95 years). In terms of patient comorbidities: $22 \%$ were diabetic $(\mathrm{n}=52)$; $18 \%$ were smokers $(\mathrm{n}=43)$; and $8 \%$ were obese (BMI $\left.>30 \mathrm{~kg} / \mathrm{m}^{2}, \mathrm{n}=19\right)$.

The mode of referral for a CT scan was an emergency admission under the surgical team in the majority of cases $(n=189)$. Other modes of referral for CT were outpatient attendances under the surgical team $(n=26)$, emergency admission under other teams $(\mathrm{n}=9)$, outpatient attendances under other teams $(n=4)$, and unknown routes $(n=7)$. The sigmoid colon was the most common site of acute diverticulitis ( $\mathrm{n}=213)$, followed by the descending colon $(\mathrm{n}=16)$, then other colonic sites ( $n=6)$. In terms of diverticulitis severity, the CT-determined Hinchey grade was classified as 1, 2, 3, or 4 in $183,29,9$, and 14 cases, respectively.

\section{Management and Outcome}

Acute diverticulitis was most commonly managed medically $(n=183)$. A smaller number of patients underwent radiological treatment in the form of percutaneous drainage $(n=4)$ and others underwent surgery, most commonly a Hartmann's procedure (Table 1). Patients managed conservatively (medically with or without radiological treatment) had a reduced length of hospital stay compared to patients managed surgically: median length of stay was 4 days (range, 3-6 days) versus 13 days (range, 8-20 days). The mortality rate in patients managed conservatively was $4.3 \%$ and for those managed surgically, $5.1 \%$.

The CT report was confident of the diagnosis of diverticulitis in 175 cases (75\%). The CT report contained a statement advising that malignancy could not be excluded in 59 cases (25\%) or that endoscopic confirmation of the diagnosis was required in 44 cases (19\%).

Five of the 235 patients with CT-proven diverticulitis were

Table 1. The Management of Patients with CT-Proven Diverticulitis, with Associated Length of Hospital Stay and Mortality

\begin{tabular}{lccc}
\hline & Patient management & Length of stay (day) & Mortality \\
\hline Medical management only & $183(78)$ & $4(3-6)$ & $10(4.3)$ \\
Interventional radiology & $4(2)$ & $8(5-12)$ & 0 \\
Laparoscopy and lavage & $4(2)$ & $12(10-14)$ & $1(0.4)$ \\
Hartmann's procedure & $24(10)$ & $13(8-20)$ & $1(0.4)$ \\
Other operative procedure & $7(3)$ & $10(8-25)$ & 0 \\
Unknown & $13(6)$ & - & 0 \\
Total & $235(100)$ & - & $12(5.1)$ \\
\hline
\end{tabular}

Values are presented as number (\%) or median (IOR). 
Table 2. Colorectal Cancer Cases

\begin{tabular}{ccl}
\hline Stage & CT confident of diverticulitis diagnosis & \multicolumn{1}{c}{ Method of CRC diagnosis } \\
\hline T4N2M0 & No & Diagnosed on follow-up endoscopy \\
T3N1M0 & Yes & Surgery during index admission \\
T4N0M0 & No & Follow-up CT demonstrated failure of inflammatory changes to resolve \\
M1 & No & Follow-up CT demonstrated liver metastases with primary sigmoid neoplasm \\
T3N2M0 & No & Surgery during index admission \\
\hline
\end{tabular}

$\mathrm{CRC}$, colorectal cancer.

registered as having CRC in the National Cancer Registry (Table 2). This represents an overall CRC prevalence of $2.1 \%$. CRC was confirmed for two of these patients by surgical resection during emergency admission, and both underwent a Hartmann's procedure. CRC was confirmed for two patients by repeat CT imaging: one patient underwent a Hartmann's procedure as the CT was suspicious of CRC with localized perforation; one patient was diagnosed with new liver metastasis, likely CRC primary, and declined further intervention. CRC was confirmed in the fifth patient by endoscopy. The CT report was not confident of the diagnosis of diverticulitis in four of the five cases of CRC. A CT report not confident of diverticulitis was significantly associated with the subsequent diagnosis of CRC ( $P=0.02$, Fisher exact test).

Patients with CT-proven diverticulitis who were managed surgically did not require follow-up endoscopy to confirm the diagnosis. Therefore, the mantra for follow-up endoscopy applied to the 187 patients managed conservatively. In this group of 187 patients, three cases of CRC were detected (prevalence of 1.6\%): one case was detected by endoscopy and two cases by repeat CT imaging.

\section{Follow-Up Endoscopy}

Follow-up lower gastrointestinal endoscopy was performed in 100 of the total 235 patients (43\%) and 90 of the 187 conservatively managed patients (48\%). Patients managed conservatively were more likely to undergo follow-up endoscopy than those managed surgically ( $48 \%$ vs. $18 \%$ ). The median time to follow-up endoscopy was 45 days (IQR, 27-78). Of the 187 patients managed conservatively, $97 \mathrm{did}$ not undergo follow-up endoscopy. This may be explained by patients undergoing prior lower gastrointestinal endoscopy in the preceding 3 years $(\mathrm{n}=23)$, prior barium enema in the preceding 3 years $(n=8)$, or repeat CT imaging $(n=7)$. Thus, 59 conservatively managed patients remained who did not undergo follow-up endoscopy for reasons that were unclear from the hospital notes. For the 100 patients who underwent
Table 3. Findings of Follow-Up Endoscopy in Patients with CT-Proven Diverticulitis

\begin{tabular}{lc}
\hline & No. (\%) \\
\hline Endoscopy findings & $19(19)$ \\
Normal examination & $75(75)$ \\
Diverticulosis & $2(2)$ \\
Stricture & $3(3)$ \\
IBD & $14(14)$ \\
Polyps & $1(1)$ \\
Malignancy & \\
Polyp details & $4(27)$ \\
Hyperplastic & $2(14)$ \\
Inflammatory & $8(57)$ \\
Low-grade adenoma & 0 \\
High-grade adenoma & $14(100)$ \\
Total &
\end{tabular}

follow-up endoscopy, colonoscopy was performed for 30 patients and flexible sigmoidoscopy for 70 patients.

\section{Endoscopy Findings}

The majority of patients who underwent follow-up endoscopy were found to have diverticulosis alone (Table 3). One patient was found to have CRC. In this case, the CT reported likely sigmoid diverticulitis with a collection but advised that CRC could not be excluded. A sigmoid CRC was detected on flexible sigmoidoscopy and biopsy confirmed an adenocarcinoma. This was defined as a pT4 N2 M0 tumor by histopathological assessment following resection (Table 2). Polyps were detected in 14 cases; eight of these were low-grade adenomas. The adenoma detection rate was, therefore, $4.3 \%$ for conservatively managed patients and $8.0 \%$ for patients undergoing follow-up endoscopy. The median size of adenomatous polyps was $5.0 \mathrm{~mm}$ (IQR, 4.5-6.3). The CT report 
Table 4. Comparison of Endoscopy Quality Indicators between Patients with CT-Proven Diverticulitis and Controls

\begin{tabular}{|c|c|c|c|c|c|c|c|}
\hline & \multicolumn{3}{|c|}{ Diverticulitis patients } & \multicolumn{3}{|c|}{ Control patients } & $P$-value \\
\hline \multicolumn{8}{|l|}{ Colonoscopies } \\
\hline Moderate-to-severe pain & $16(53)$ & $10(13)$ & $4(33)$ & $8,184(50)$ & $1,309(8)$ & $6,958(42)$ & 0.630 \\
\hline Complete procedure & $2(7)$ & 28 (93) & 0 & 1,385 (8) & $15,066(92)$ & 0 & 0.990 \\
\hline Duration (min) & $22(14-33)$ & \multicolumn{5}{|c|}{$27(19-37)$} & 0.060 \\
\hline Moderate-to-severe pain & $9(14)$ & $20(31)$ & $36(55)$ & $1,577(17)$ & $1,230(14)$ & $6,297(69)$ & 0.010 \\
\hline Complete procedure & $28(43)$ & $37(57)$ & 0 & $2,478(27)$ & $6,626(73)$ & 0 & 0.006 \\
\hline Duration (min) & $13(9-18)$ & \multicolumn{5}{|c|}{$11(8-16)$} & $<0.050$ \\
\hline
\end{tabular}

Values are presented number (\%) or median (IOR).

advised that CRC could not be excluded or recommended endoscopic evaluation in four of the eight cases of low-grade adenomas.

\section{Endoscopy Quality Indicators}

The completion rate, discomfort, and duration of colonoscopies and flexible sigmoidoscopies for patients with CT-proven diverticulitis were compared with all other procedures performed over the same time period at the Trust (Table 4). The extent of the procedure (completion) was available for all colonoscopies and flexible sigmoidoscopies. The discomfort level was available for $66 \%$ of the colonoscopies and $45 \%$ of the flexible sigmoidoscopies. The duration was available for $91.5 \%$ of the colonoscopies and $81 \%$ of the flexible sigmoidoscopies. Flexible sigmoidoscopies were more often incomplete, caused more discomfort, and were of longer duration for patients with CT-proven diverticulitis compared with controls. These differences were statistically significant.

\section{DISCUSSION}

Traditionally, it is an accepted practice to perform an endoscopic evaluation of the colon either by a sigmoidoscopy or colonoscopy following acute diverticulitis to exclude an underlying malignancy. ${ }^{10}$ This practice is still recommended in U.K. and U.S. guidelines ${ }^{3,5}$ but adds to the cost of diverticulitis management, expends endoscopy resources, and involves some risk to patients, including bowel perforation. The management of acute diverticulitis has rapidly changed over the last 20 years with the greater use of CT to confirm the diagnosis and a trend towards conservative management of the disease rather than surgical resection. ${ }^{9}$ The widespread availability of high-resolution CT scanners means that the colon can now be evaluated in great detail in the acute setting, confidently diagnosing acute diverticulitis and its complications. CT has a sensitivity and specificity approaching 99\% for the diagnosis of diverticulitis. ${ }^{11,12}$ Thus the question arises whether routine colonic examination to exclude underlying CRC following a CT-proven diagnosis of acute diverticulitis is necessary.

In our study, we identified 235 patients presenting to our Trust with a CT-proven diagnosis of acute diverticulitis. Eighty-six percent of patients were managed conservatively. Follow-up endoscopy was performed for $48 \%$ of patients managed conservatively, a smaller proportion than probably expected. Studies of follow-up endoscopy in patients with CT-proven diverticulitis have reported much higher rates of $83 \%^{8}$ and $76 \%{ }^{13}$ consistent with accepted practice. However, other studies have reported lower rates of $29 \%,{ }^{10} 39 \%,{ }^{14}$ and $46 \%{ }^{7}$ consistent with our findings and perhaps reflecting an appreciation of the low yield of endoscopy in the current era of CT-led diagnosis. Some of our patients had undergone previous lower gastrointestinal endoscopy or barium enema investigations, obviating the need for follow-up endoscopy. Nevertheless, there were still 59 patients for whom no explanation for foregoing follow-up endoscopy could be found. This may simply reflect doctor or patient choice or it is possible that such patients may have previously undergone, or planned to undergo endoscopic follow-up at another hospital. In support of patient choice partly explaining incomplete endoscopic follow-up, previous studies have shown an appreciable endoscopy cancellation rate by patients of between $8 \%$ and $20 \%{ }^{8,13}$

The CRC detection rate from follow-up endoscopy in our 
study was one case in 100 patients (1\%). A systematic review addressed this question in 2012, assessing 10 studies (771 patients) and reporting a CRC detection rate of $2.1 \% .{ }^{6}$ However, only four of the 10 studies specifically investigated colonoscopy follow-up, and one of these four evaluated the use of CT colonography rather than contrast-enhanced CT of the abdomen and pelvis.

Two more recent studies found that no CRC was detected in 100 and 205 follow-up colonoscopies for CT-proven diverticulitis. ${ }^{7,15}$ The most recent review on this subject included these two studies. ${ }^{9}$ Similarly, a study of 80 follow-up endoscopies detected no CRC. ${ }^{8}$ However, three recent large studies reported higher CRC detection rates of $2.1 \%,{ }^{10} 4.3 \%$, ${ }^{16}$ and $2.2 \%$. ${ }^{14}$ These studies followed up 319, 394, and 402 patients by endoscopy, respectively, the results of which are similar to the overall CRC detection rate in our study. A Dutch study compared the CRC detection rate in patients with CT-proven uncomplicated diverticulitis using average risk participants of a CRC screening trial. ${ }^{17} \mathrm{CRC}$ was detected in five of 401 diverticulitis patients (1.2\%) versus nine of 1,426 screening patients $(0.6 \%)$. The calculated OR was not statistically significant, leading the investigators to recommend the omission of routine endoscopy following uncomplicated diverticulitis. However, the trial diverticulitis patients who completed follow-up may have had a lower CRC prevalence than those who did not complete follow-up and trial patients as a whole may not represent all diverticulitis patients, as identified in retrospective studies. Furthermore, endoscopy for the screening patients was provided by expert screening colonoscopists compared with general hospital endoscopists for the diverticulitis group-a factor that may translate into lower pathology detection rates in the diverticulitis group.

A further recent study of 249 conservatively-managed diverticulitis patients who underwent follow-up colonoscopy reported a CRC detection rate of $1.6 \%$ and supported the view that routine follow-up by colonoscopy may not be necessary. ${ }^{18}$

A meta-analysis of studies investigating the prevalence of CRC in 68,324 asymptomatic individuals screened by colonoscopy reported a CRC detection rate of $0.78 \%$. Advanced adenomas were found in $5.0 \%$ cases. ${ }^{19}$ Advanced adenomas were defined as adenomas of greater than 10-mm diameter, with high-grade dysplasia, or with more than $25 \%$ villous architecture. Similar advanced adenoma detection rates of $5 \%$ to $8 \%$ have been reported in diverticulitis patients. ${ }^{8,17,18} \mathrm{In}$ our study, no advanced adenomas were detected. The overall adenoma detection rate in our study was $8.0 \%$ for those patients that underwent follow-up endoscopy. This rate is entirely in keeping with expected rates from screening studies. $^{19}$

The CRC detection rate from our study is comparable with that from screened asymptomatic populations. However, less than half of our patients underwent follow-up endoscopy. To address this issue, the prevalence of CRC in our total population of 235 patients was sought using the National Cancer Registry. Our overall CRC prevalence of $2.1 \%$ is significantly higher than that of an asymptomatic screened population, as is the prevalence in conservatively managed patients (1.6\%).

Unfortunately, there are no accepted criteria to guide the need for colonoscopy in patients with acute diverticulitis. Variations in CRC detection rate may, therefore, reflect different thresholds for reporting CT findings as acute diverticulitis. In our study, we investigated the confidence of CT reporting in acute diverticulitis and found that reports stated CRC could not be excluded or recommended endoscopic evaluation in $25 \%$ cases. The CT reports of four of the five proven cases of CRC stated that CRC could not be excluded by CT. Restricting follow-up endoscopy to the minority of patients with CT reports that are not confident of the diagnosis of acute diverticulitis could possibly reduce the overall burden of endoscopic follow-up whilst maximizing the CRC detection rate. Two studies tested the association of specific CT findings with the presence of CRC on later endoscopy. One of these studies showed that CT findings of an abscess, perforation, or fistulae were associated with $\mathrm{CRC}^{10}$ while the other study found that a localized mass, large bowel obstruction, or significant retroperitoneal lymphadenopathy were associated with CRC. ${ }^{14}$

Acute diverticulitis and CRC can be difficult to differentiate on CT imaging. Both may demonstrate features of colonic wall thickening, varying degrees of inflammation, and signs of obstruction. ${ }^{20}$ Perforated CRC may also mimic perforated diverticulitis. In general, colonic wall thickening is greater and more often eccentric in CRC, while pericolonic fat stranding and long segment involvement are more typical of acute diverticulitis. ${ }^{21}$ Overlap of CT features renders specific diagnostic criteria impossible and a CT diagnosis of acute diverticulitis is, therefore, inherently subjective. We, thus, propose that for practical purposes, endoscopic followup should be conducted in cases of radiological uncertainty and that CT reports of acute diverticulitis should be accompanied by a statement of diagnostic certainty. Despite this, one patient in our study was diagnosed with CRC having undergone a CT scan which was confident of the diagnosis 
of diverticulitis. For this reason, we suggest that some form of radiological follow-up, such as repeat CT imaging or CT colonography, should be performed for all patients not selected for endoscopy.

It is well established that diverticulosis is a risk factor for difficult colonoscopy, reflected in poor completion rates, ${ }^{22}$ significant discomfort, and prolonged procedural duration. ${ }^{23}$ Diverticulosis is a common finding and it is perhaps more likely to be reported as a justification for incompletion in difficult cases. In our study, we compared various quality indicators (completion rates, discomfort levels, and procedure duration) of lower gastrointestinal endoscopies between our population of patients with CT-proven diverticulitis, rather than diverticulosis, with a control group consisting of all patients that underwent the same procedure at our Trust over the same time period. To the authors' knowledge, such a comparison has not been reported previously in the literature. There was no difference in any of the quality indicators for colonoscopies. This result may be surprising, but colonoscopies formed the minority of lower gastrointestinal endoscopy follow-up investigations. Flexible sigmoidoscopies were the chosen investigation in $70 \%$ cases and were more often incomplete, caused more discomfort, and were of longer duration compared with controls. Our results, therefore, indicate that performing follow-up flexible sigmoidoscopy in patients with CT-proven diverticulitis subjects these patients to a more painful, prolonged, and ineffective investigation compared with the average patient.

The most important limitations of this study are its retrospective design and small sample size. A prospective study would provide consistent follow-up and make use of structured CT reports with a mandatory statement on the diagnostic certainty of acute diverticulitis. Nevertheless, the use of data from the National Cancer Registry makes identification of CRC cases in our cohort robust. Only five cases of CRC were identified and a more accurate measurement of the true prevalence of CRC in this patient group could only be estimated by a much larger cohort of patients, requiring a multicenter study-this would also allow for investigation of differences in local policies regarding follow-up.

In conclusion, this study has demonstrated low but significant rates of CRC detection by follow-up endoscopy in patients with CT-proven diverticulitis, consistent with previously published studies. Importantly, the prevalence of CRC in patients with CT-proven diverticulitis was greater than that expected for a screened asymptomatic population. Therefore, follow-up by imaging or endoscopy is required. In addition, we have shown that CT reports contained a state- ment of diagnostic uncertainty in $25 \%$ cases. We suggest that such a report mandates follow-up endoscopy but in other cases, follow-up imaging may be preferred. Furthermore, follow-up endoscopy in patients with CT-proven diverticulitis is associated with patient discomfort and higher rates of incompletion.

\section{ACKNOWLEDGEMENTS}

The authors would like to thank Christopher Roberts (PACS Team, Sandwell \& West Birmingham NHS Trust) for his help in interrogating the PACS database and John Broggio (Knowledge \& Intelligence Team, Public Health England) for his help in searching the National Cancer Registry.

\section{REFERENCES}

1. Hughes LE. Postmortem survey of diverticular disease of the colon: I. diverticulosis and diverticulitis. Gut 1969;10:336-344.

2. Matrana MR, Margolin DA. Epidemiology and pathophysiology of diverticular disease. Clin Colon Rectal Surg 2009;22:141-146.

3. Fozard JB, Armitage NC, Schofield JB, Jones OM; Association of Coloproctology of Great Britain and Ireland. ACPGBI position statement on elective resection for diverticulitis. Colorectal Dis 2011;13 Suppl 3:1-11.

4. Colinic diverticular disease: commissioning guide. Royal College of Surgeons Web site. https://www.rcseng.ac.uk/libraryand-publications/college-publications/docs/colinic-diverticular-disease/. Accessed February 23, 2017.

5. Sai VF, Velayos F, Neuhaus J, Westphalen AC. Colonoscopy after CT diagnosis of diverticulitis to exclude colon cancer: a systematic literature review. Radiology 2012;263:383-390.

6. Schmilovitz-Weiss H, Yalunin E, Boaz M, et al. Does a colonoscopy after acute diverticulitis affect its management? A single center experience. J Clin Gastroenterol 2012;46:317-320.

7. Page AA, Khan A, Rajaratnam S, Carroll N, Hall NR, Davies RJ. Is follow-up endoscopy necessary after successful medical treatment of CT-proven acute diverticulitis? Gut 2011;60(Suppl 1):A72.

8. Sharma PV, Eglinton T, Hider P, Frizelle F. Systematic review and meta-analysis of the role of routine colonic evaluation after radiologically confirmed acute diverticulitis. Ann Surg 2014;259:263-272.

9. Lau KC, Spilsbury K, Farooque Y, et al. Is colonoscopy still mandatory after a CT diagnosis of left-sided diverticulitis: can colorectal cancer be confidently excluded? Dis Colon Rectum 2011;54:1265-1270. 
10. Rao PM, Rhea JT, Novelline RA, et al. Helical CT with only colonic contrast material for diagnosing diverticulitis: prospective evaluation of 150 patients. AJR Am J Roentgenol 1998;170:14451449.

11. Westwood DA, Eglinton TW, Frizelle FA. Routine colonoscopy following acute uncomplicated diverticulitis. Br J Surg 2011;98:1630-1634.

12. Lahat A, Yanai H, Sakhnini E, Menachem Y, Bar-Meir S. Role of colonoscopy in patients with persistent acute diverticulitis. World J Gastroenterol 2008;14:2763-2766.

13. Elmi A, Hedgire SS, Pargaonkar V, Cao K, McDermott S, Harisinghani M. Is early colonoscopy beneficial in patients with CTdiagnosed diverticulitis? AJR Am J Roentgenol 2013;200:12691274.

14. van de Wall BJ, Reuling EM, Consten EC, et al. Endoscopic evaluation of the colon after an episode of diverticulitis: a call for a more selective approach. Int J Colorectal Dis 2012;27:11451150

15. Sallinen V, Mentula P, Leppäniemi A. Risk of colon cancer after computed tomography-diagnosed acute diverticulitis: is routine colonoscopy necessary? Surg Endosc 2014;28:961-966.

16. Daniels L, Ünlü Ç, de Wijkerslooth TR, et al. Yield of colonoscopy after recent CT-proven uncomplicated acute diverticulitis: a comparative cohort study. Surg Endosc 2015;29:2605-2613.
17. Brar MS, Roxin G, Yaffe PB, Stanger J, MacLean AR, Buie WD. Colonoscopy following nonoperative management of uncomplicated diverticulitis may not be warranted. Dis Colon Rectum 2013;56:1259-1264.

18. Niv Y, Hazazi R, Levi Z, Fraser G. Screening colonoscopy for colorectal cancer in asymptomatic people: a meta-analysis. Dig Dis Sci 2008;53:3049-3054.

19. Goh V, Halligan S, Taylor SA, Burling D, Bassett P, Bartram CI. Differentiation between diverticulitis and colorectal cancer: quantitative CT perfusion measurements versus morphologic criteria--initial experience. Radiology 2007;242:456-462.

20. Destigter KK, Keating DP. Imaging update: acute colonic diverticulitis. Clin Colon Rectal Surg 2009;22:147-155.

21. Dafnis G, Granath F, Påhlman L, Ekbom A, Blomqvist P. Patient factors influencing the completion rate in colonoscopy. Dig Liver Dis 2005;37:113-118.

22. Elphick DA, Donnelly MT, Smith KS, Riley SA. Factors associated with abdominal discomfort during colonoscopy: a prospective analysis. Eur J Gastroenterol Hepatol 2009;21:1076-1082.

23. Anderson JC, Messina CR, Cohn W, et al. Factors predictive of difficult colonoscopy. Gastrointest Endosc 2001;54:558-652. 